\title{
Radiative hydrodynamics simulations of red supergiant stars
}

\section{Simulations of convection on Betelgeuse match interferometric observations}

\author{
A. Chiavassa ${ }^{1,2}$, X. Haubois ${ }^{3}$, J. S. Young ${ }^{4}$, B. Plez ${ }^{2}$, E. Josselin² ${ }^{2}$, G. Perrin ${ }^{3}$, and B. Freytag 5,6 \\ 1 Max-Planck-Institut für Astrophysik, Karl-Schwarzschild-Str. 1, Postfach 1317, 85741 Garching b. München, Germany \\ e-mail: chiavass@mpa-garching.mpg.de \\ 2 GRAAL, Université de Montpellier II - IPM, CNRS, Place Eugéne Bataillon, 34095 Montpellier Cedex 05, France \\ 3 Observatoire de Paris, LESIA, UMR 8109, 92190 Meudon, France \\ 4 Astrophysics Group, Cavendish Laboratory, JJ Thomson Avenue, Cambridge CB3 0HE, UK \\ 5 Centre de Recherche Astrophysique de Lyon, UMR 5574: CNRS, Université de Lyon, École Normale Supérieure de Lyon, \\ 46 allée d'Italie, 69364 Lyon Cedex 07, France \\ 6 Department of Physics and Astronomy, Division of Astronomy and Space Physics, Uppsala University, Box 515, 75120 Uppsala, \\ Sweden
}

Received 18 December 2009 / Accepted 2 March 2010

\section{ABSTRACT}

\begin{abstract}
Context. The red supergiant (RSG) Betelgeuse is an irregular variable star. Convection may play an important role in understanding this variability. Interferometric observations can be interpreted using sophisticated simulations of stellar convection.

Aims. We compare the visibility curves and closure phases obtained from our 3D simulation of RSG convection with CO5BOLD to various interferometric observations of Betelgeuse from the optical to the $H$ band to characterize and measure the convection pattern on this star.

Methods. We use a 3D radiative-hydrodynamics (RHD) simulation to compute intensity maps in different filters and thus derive interferometric observables using the post-processing radiative transfer code OPTIM3D. The synthetic visibility curves and closure phases are compared to observations.

Results. We provide a robust detection of the granulation pattern on the surface of Betelgeuse in both the optical and the $H$ band based on excellent fits to the observed visibility points and closure phases. We determine that the Betelgeuse surface in the $H$ band is covered by small to medium scale (5-15 mas) convection-related surface structures and a large $(\approx 30$ mas $)$ convective cell. In this spectral region, $\mathrm{H}_{2} \mathrm{O}$ molecules are the main absorbers and contribute to both the small structures and the position of the first null of the visibility curve (i.e., the apparent stellar radius).
\end{abstract}

Key words. stars: individual: Betelgeuse - stars: atmospheres - hydrodynamics - radiative transfer - techniques: interferometric

\section{Introduction}

Betelgeuse is a red supergiant star (Betelgeuse, HD 39801, M1-2Ia-Ibe) and is one of the brightest stars in the optical and near infrared. This star exhibits variations in integrated brightness, surface features, and the depths, shapes, and Doppler shifts of its spectral lines. Visual-wavelength observations of its brightness cover almost a hundred years. The irregular fluctuations of its light curve are clearly aperiodic and resemble a series of outbursts. Kiss et al. (2006) studied the variability of different red supergiant (RSG) stars including Betelgeuse and found a strong noise component in the photometric variability, probably caused by the large convection cells. In addition to this, the spectral line variations have been analyzed by several authors, who inferred the presence of large granules and high convective velocities (Josselin \& Plez 2007; Gray 2008). Gray also found line bisectors that have predominantly reversed $\mathrm{C}$-shapes, and line shape variations occurring at the $1 \mathrm{~km} \mathrm{~s}^{-1}$ level that have no obvious connection to their shifts in wavelength.

The position of Betelgeuse on the H-R diagram is highly uncertain, because of the uncertainty in its effective temperature. Levesque et al. (2005) used one-dimensional MARCS models (Gustafsson et al. 2003, 1975) to fit the incredibly rich $\mathrm{TiO}$ molecular bands in the optical region of the spectrum for several RSGs. They found an effective temperature of $3650 \mathrm{~K}$ for Betelgeuse. Although they obtained good agreement with the evolutionary tracks, problems remain. There is a mismatch in the IR colors that could be due to atmospheric temperature inhomogeneities characteristic of convection (Levesque et al. 2006). The distance of Betelgeuse also has large uncertainties because of errors related to the positional movement of the stellar photocenter. Harper et al. (2008) derived a distance of (197 \pm 45 pc) using high spatial resolution, multiwavelength, VLA radio positions combined with Hipparcos Catalogue Intermediate Astrometric Data.

Betelgeuse is one of the most well studied RSGs in term of multiwavelength imaging because of its high luminosity and large angular diameter. The existence of hot spots on its surface has been proposed to explain numerous interferometric observations with WHT and COAST (Buscher et al. 1990; Wilson et al. 1992; Tuthill et al. 1997; Wilson et al. 1997; Young et al. 2000, 2004) that detected time-variable inhomogeneities in the brightness distribution. These authors fitted the visibility and closure phase data with a circular limb-darkened disk and zero to three spots. A large spot was detected by Uitenbroek et al. (1998) with HST. The non-spherical shape of Betelgeuse was also detected by Tatebe et al. (2007) in the mid-infrared. 
Haubois et al. (2009) published a reconstructed image of Betelgeuse in the $H$ band with two spots using the same data as presented in this work. Kervella et al. (2009) resolved Betelgeuse using diffraction-limited adaptive optics in the nearinfrared and found an asymmetric envelope around the star with a bright plume extending in the southwestern region. They claimed the plume was either due to the presence of a convective hot spot or caused by stellar rotation. Ohnaka et al. (2009) presented VLTI/AMBER observations of Betelgeuse at high spectral resolution and spatially resolved $\mathrm{CO}$ gas motions. They claimed that these motions were related to convective motions in the upper atmosphere or to intermittent mass ejections in clumps or arcs.

Radiation hydrodynamics (RHD) simulations of red supergiant stars are available (Freytag et al. 2002) to interpret past and future observations. Chiavassa et al. (2009), hereafter Paper I, used these simulations to explore the impact of the granulation pattern on observed visibility curves and closure phases and detected a granulation pattern on Betelgeuse in the $K$ band by fitting the existing interferometric data of Perrin et al. (2004).

This paper is the second in a series aimed at exploring the convection in RSGs. The main purpose is to compare RHD simulations to high-angular resolution observations of Betelgeuse covering a wide spectral range from the optical region to the near-infrared $H$ band, to confirm the detection of convective cells on its surface.

\section{3D radiation-hydrodynamics simulations and post-processing radiative transfer}

We employed numerical simulations obtained using $\mathrm{CO}^{5} \mathrm{BOLD}$ (Freytag et al. 2002; Freytag 2003; Freytag \& Höfner 2008) and in particular the model st $35 \mathrm{gm} 03 \mathrm{n} 07$ that was deeply analyzed in Paper I. The model has a mass of $12 M_{\odot}$, a numerical resolution of $235^{3}$ grid points with a step of $8.6 R_{\odot}$, an average luminosity over spherical shells and over time of $L=93000 \pm 1300 L_{\odot}$, an effective temperature of $T_{\text {eff }}=3490 \pm 13 \mathrm{~K}$, a radius of $R=$ $832 \pm 0.7 R_{\odot}$, and surface gravity $\log (g)=-0.337 \pm 0.001$. This is our most successfull RHD simulation so far because it has stellar parameters closest to Betelgeuse $\left(T_{\text {eff }}=3640 \mathrm{~K}\right.$, Levesque et al. 2005 and $\log (g)=-0.3$, Harper et al. 2008).

We used the 3D pure-LTE radiative transfer code OPTIM3D described in Paper I to compute intensity maps from all the suitable snapshots of the 3D hydrodynamical simulation. The code takes into account the Doppler shifts caused by the convective motions. The radiation transfer is calculated in detail using pre-tabulated extinction coefficients generated with the MARCS code (Gustafsson et al. 2008). These tables are functions of temperature, density and wavelength, and were computed for the solar composition of Asplund et al. (2006). The tables include the same extensive atomic and molecular data as the MARCS models. They were constructed with no micro-turbulence broadening and the temperature and density distributions are optimized to cover the values encountered in the outer layers of the RHD simulations.

\section{Observations}

The data presented in this work were acquired by two independent groups with different telescopes and they cover a large wavelength range from the optical to the near infrared. The log of the observations is reported in Table 1.
Table 1. Log of the observations.

\begin{tabular}{ccc}
\hline \hline Date & Telescope & Filter $($ central $\lambda)$ \\
\hline October 7, 2005 & IOTA & IONIC -16000 \\
October 8, 2005 & IOTA & IONIC -16000 \\
October 10, 2005 & IOTA & IONIC -16000 \\
October 11, 2005 & IOTA & IONIC -16000 \\
October 12, 2005 & IOTA & IONIC -16000 \\
October 16, 2005 & IOTA & IONIC -16000 \\
October 21, 1997 & COAST & $12900 \AA$ \\
October 24, 1997 & COAST & $9050 \AA$ \\
October 31, 1997 & COAST & $9050 \AA$ \\
November 11, 1997 & COAST & $12900 \AA$ \\
November 12,1997 & COAST & $9050 \AA$ \\
November 15, 1997 & WHT & $7000 \AA$ \\
November 16, 1997 & WHT & $9050 \AA$ \\
November 21, 1997 & COAST & $9050 \AA$ \\
January 29, 2004 & COAST & $7500,7820,9050 \AA$ \\
February 8, 2004 & COAST & $7500,7820,9050 \AA$ \\
February 25, 2004 & COAST & $7500,7820,9050 \AA$ \\
February 29, 2004 & COAST & $7500,7820,9050 \AA$ \\
March 1, 2004 & COAST & $7500,7820,9050 \AA$ \\
March 2, 2004 & COAST & $7500,7820,9050 \AA$ \\
\hline
\end{tabular}

\subsection{Data at $16400 \AA$}

The $H$ band data were acquired with the 3 telescope interferometer IOTA (Infrared Optical Telescope Array, Traub et al. 2003) located at Mount Hopkins in Arizona. Light collected by three apertures (siderostats of $0.45 \mathrm{~m}$ diameter) was spatially filtered by single mode fibers to clean the wavefronts, removing high frequency atmospheric corrugations that affect the fringe contrast. The beams were then combined with IONIC (Berger et al. 2003). This integrated optics component combines 3 input beams in a pairwise manner. Fringes were encoded in the time domain using piezo-electric path modulators, and detected with a near-infrared camera utilizing a PICNIC detector (Pedretti et al. 2004).

Betelgeuse was observed in the $H$ band $(16400 \pm 1000 \AA$, Fig. 1) on 6 nights between Oct. 7, 2005 and Oct. 16, 2005. Five different configurations of the interferometer telescopes were used, to cover a wide range of spatial frequencies between 12 and $95 \operatorname{arcsec}^{-1}$. To calibrate the instrumental transfer function, observations of Betelgeuse were interleaved with observations of a reference (calibrator) star, HD 36167.

Data reduction was carried out using an IDL pipeline (Monnier et al. 2004; Zhao et al. 2007). To measure the closure phase, we took the phase of the complex triple product (bispectrum, Baldwin et al. 1986). The instrumental closure phase of IONIC3 drifted by less than 1 degree over many hours owing to the miniature dimensions of the integrated optics component. For both the squared visibilities and the closure phase, the random errors were calculated with the bootstrap technique, in which a statistic is repeatedly re-estimated by Monte-Carlo sampling the original data with replacement. Full details of the observations and data reduction can be found in Haubois et al. (2009).

\subsection{Data from 7000 to $12900 \AA$}

For this wavelength range, we used data taken at two different epochs. The observations carried out in 1997 (Young et al. 2000) were acquired with the Cambridge Optical Aperture Synthesis Telescope (COAST) on baselines up to $8.9 \mathrm{~m}$ (with central wavelengths/bandwidths of 9050/500 and 12900/1500 $\AA$ ) and 
$\mathrm{TiO}$
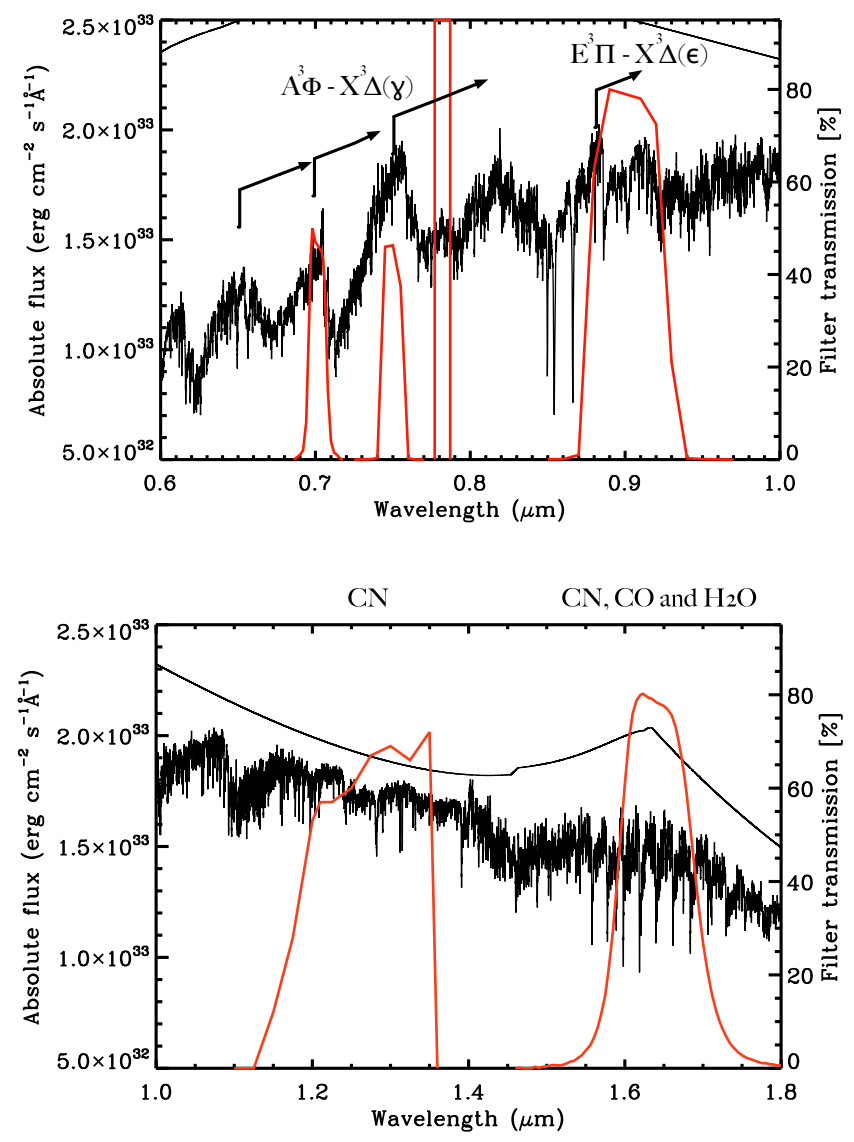

Fig. 1. Transmission curves (red) of the bandpass filters used for Betelgeuse observations at COAST and WHT: central wavelengths at 7000, 7500, 7820, and $12900 \AA$ and nominal bandwidths $(F W H M)$ of $100,130,50,500$, and $1500 \AA$, respectively (Young et al. 2004, 2000) (the filter curve for the narrow bandpass centered on $7820 \AA$ has been lost hence we assumed a top-hat filter); and for Betelgeuse observations at IOTA: $16400 \AA$ with $1000 \AA$ bandwidth (Haubois et al. 2009). The black line is the synthetic spectrum with its continuum computed from the RHD simulation snapshot of Fig. 8; the molecules that contribute the most in every filter have been highlighted.

by non-redundant aperture masking with the William Herschel Telescope (WHT) on baselines up to $3.7 \mathrm{~m}$ (with central wavelengths/bandwidths of 7000/100 and 9050/500). The observations carried out in 2004 were obtained with COAST on baselines up to $6.1 \mathrm{~m}$ (bandpasses 7500/130, 7820/50, and 9050/500 ̊). Figure 1 shows all the filters used.

\subsubsection{COAST data from 1997}

The COAST data were acquired during October and November 1997. Observations at $9050 \AA$ were made using the standard beam-combiner and avalanche photodiode detectors (Baldwin et al. 1994), while $12900 \AA$ observations were obtained with a separate pupil-plane combiner optimized for JHK bands (Young et al. 1998).

Observations of Betelgeuse were interleaved with observations of calibrator stars, either unresolved or of small and known diameters. If at least three baselines were measurable and the atmospheric coherence time was sufficiently long, closure phase measurements were also collected, by recording fringes on three baselines simultaneously.

Data reduction was carried out using standard methods in which the power spectrum and bispectrum of the interference fringes were averaged over each dataset (Burns et al. 1997). The resulting visibilities had formal fractional errors in the range $2-10 \%$ of the values, and the closure phases had typical uncertainties of $5-10^{\circ}$. Additional uncertainties of $10-20 \%$ were added to the visibility amplitudes to accommodate potential changes in the seeing conditions between observations of the science target and calibrator stars.

\subsubsection{WHT data from 1997}

The observations performed with the WHT used the nonredundant aperture masking method (Baldwin et al. 1986; Haniff et al. 1987) and employed a five-hole linear aperture mask. Filters centred on both $7000 \AA$ and $9050 \AA$ were used to select the observing waveband; only the $7000 \AA$ data are presented in this paper. The resulting interference fringes were imaged onto a CCD and one-dimensional fringe snapshots were recorded at 12-ms intervals. For each orientation of the mask, the fringe data were reduced using standard procedures (Haniff et al. 1987; Buscher et al. 1990) to provide estimates of the visibility amplitudes on all 10 interferometer baselines and the closure phases on the 10 (linear) triangles of baselines. As for the COAST measurements, the uncertainties in the visibility amplitudes were dominated by calibration errors, which in this instance were unusually large (fractional error $\sim 30 \%$ ). On the other hand, the calibrated closure phase measurements had typical errors of only $1-3^{\circ}$. The orientation and scale of the detector were determined by observations of two close visual binaries with well-determined orbits.

\subsubsection{COAST data from 2004}

The observations taken in 2004 (Young et al. 2004) were acquired with COAST using the standard beam combiner and filters centered on 7500, 7820, and $9050 \AA$ of $F W H M 130,50$, and $500 \AA$, respectively. The raw interference fringe data were reduced using the same methods utilised for the 1997 COAST data to obtain a set of estimates of the visibility amplitude and closure phase for each observing waveband.

\section{Comparison of simulations and observations}

We compare the synthetic visibility curves and closure phases to the observations. For this purpose, we used all the snapshots from the RSG simulation to compute intensity maps with OPTIM3D. These maps were normalized to the filter transmissions of Fig. 1 as $\frac{\int I_{\lambda} \tau(\lambda) \mathrm{d} \lambda}{\int \tau(\lambda) \mathrm{d} \lambda}$, where $I(\lambda)$ is the intensity and $\tau(\lambda)$ is the optical transmission of the filter at a certain wavelength. For each intensity map, a discrete Fourier transform $(F T)$ was then calculated. The visibility $V$ is defined as the modulus $|z|$ of the complex Fourier transform $z=x+i y$ (where $x$ is real part of the complex number $z$ and $y$ its imaginary part) normalized to the modulus at the origin of the frequency plane $\left|z_{0}\right|$, with the phase $\theta$ defined as $\tan \theta=\mathfrak{J}(z) / \mathfrak{R}(z)$. The closure phase is defined as the phase of the triple product (or bispectrum) of the complex visibilities on three baselines, which form a closed loop joining three stations A, B, and C. If the projection 
of the baseline $\mathrm{AB}$ is $\left(u_{1}, v_{1}\right)$, that for $\mathrm{BC}$ is $\left(u_{2}, v_{2}\right)$, and thus $\left(u_{1}+u_{2}, v_{1}+v_{2}\right)$ for $\mathrm{AC}$, the closure phase is:

$$
\begin{aligned}
\phi_{C}\left(u_{1}, v_{1}, u_{2}, v_{2}\right)= & \arg \left(V\left(u_{1}, v_{1}\right) \times V\left(u_{2}, v_{2}\right)\right. \\
& \left.\times V^{*}\left(u_{1}+u_{2}, v_{1}+v_{2}\right)\right) .
\end{aligned}
$$

The projected baselines and stations are those of the observations.

Following the method explained in Paper I, we computed visibility curves and closure phases for 36 different rotation angles with a step of $5^{\circ}$ from all the available intensity maps ( $\approx 3.5$ years of stellar time), giving a total of $\approx 2000$ synthetic visibilities and $\approx 2000$ synthetic closure phases per filter.

\subsection{Data at $16400 \AA$}

We begin by comparing with the $16400 \AA$ data because this filter is centered where the $\mathrm{H}^{-1}$ continuous opacity minimum occurs. Consequently, the continuum-forming region is more visible and the granulation pattern is characterized by large-scale granules of about $400-500 R_{\odot}(\approx 60 \%$ of the stellar radius $)$ evolving on a timescale of years (Fig. 4 in Paper I). On the top of these cells, there are short-lived (a few months to one year) small-scale (about 50-100 $R_{\odot}$ ) structures. The resulting granulation pattern causes significant fluctuations in the visibility curves and the signal to be expected in the second, third, and fourth lobes deviates greatly from that predicted by uniform disk (UD) and limbdarkened disk (LD) models (Fig. 11 in Paper I). The closure phases also show large departures from 0 and $\pm \pi$, the values that would correspond to a point-symmetric brightness distribution.

Within the large number of computed visibilities and closure phases for this filter, we found that some match the observation data very well (Fig. 2). We selected the best-fit model snapshot by minimizing the function

$\chi^{2}=\frac{1}{N}\left[\sum_{i=1}^{N_{V}}\left(\frac{V_{i}-M_{V_{i}}}{\sigma_{V_{i}}}\right)^{2}+\sum_{i=1}^{N_{\phi_{C}}}\left(\frac{\phi_{C_{i}}-M_{\phi_{C_{i}}}}{\sigma_{\phi_{C_{i}}}}\right)^{2}\right]$,

where $V_{i}$ is the observed visibility amplitude data with its corresponding error $\sigma_{V_{i}}, M_{V_{i}}$ is the synthetic visibility amplitude at the same spatial frequency, $\phi_{C_{i}}$ is the observed closure phase with corresponding error $\sigma_{\phi_{C_{i}}}$, and $M_{\phi_{C_{i}}}$ the synthetic closure phase for the observed UV coordinates. The closest matching visibilities and closure phases correspond to a particular snapshot and rotation angle.

In Fig. 3, the simulation has been scaled to an apparent diameter of $\sim 45.1$ mas to fit the data points in the first lobe, corresponding to a distance of $172.1 \mathrm{pc}$ for the simulated star. The angular diameter is slightly larger than the limb-darkened diameter of $44.28 \pm 0.15$ mas found by Haubois et al. (2009). Our distance is also in agreement with Harper et al. (2008), who reported a distance of $197 \pm 45 \mathrm{pc}$. Using the distance of Harper et al. and an effective temperature of $3650 \mathrm{~K}$ (Levesque et al. 2005), the radius is $R=890 \pm 200 R_{\odot}$, neglecting any uncertainty in $T_{\text {eff }}$. On the other hand, using the distance of Harper et al. and the apparent diameter of 45 mas (Perrin et al. 2004), the radius is $R=950 \pm 200 R_{\odot}$. All these results match evolutionary tracks by Meynet $\&$ Maeder (2003) for an initial mass of between 15 and $25 M_{\odot}$. The radius $\left(R \approx 832 R_{\odot}\right.$, see Sect. 2$)$ and the effective temperature $\left(T_{\text {eff }} \approx 3490 \mathrm{~K}\right)$ of our 3D simulation are smaller because the simulations start with an initial model that has an estimated radius, a certain envelope mass, a certain potential profile, and a prescribed luminosity. However, during the run the internal structure relaxes to something not to far away from
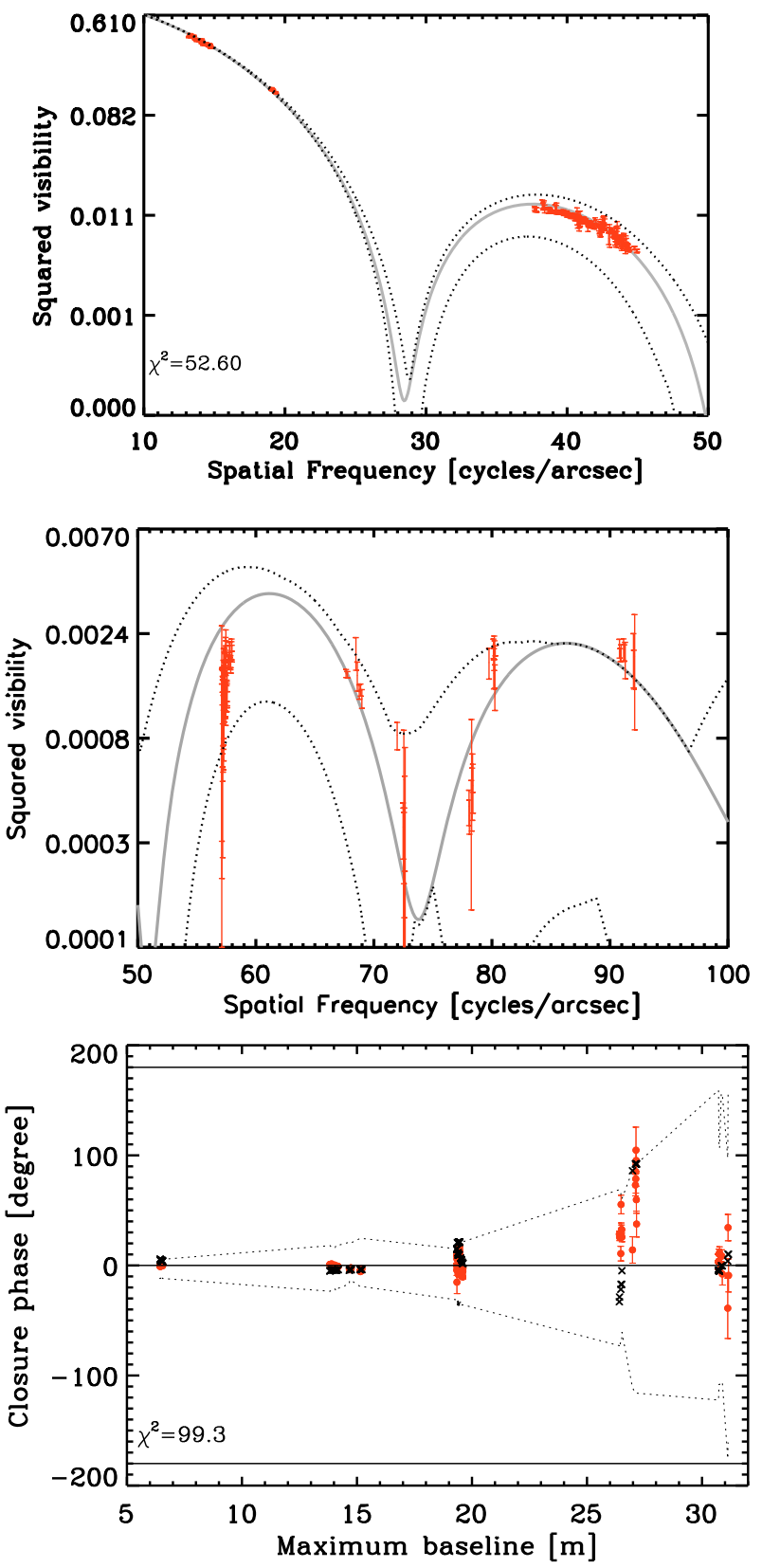

Fig. 2. Top and central panels: the best-matching synthetic squared visibility (grey) compared to the observations of Betelgeuse (Haubois et al. 2009, red). The reduced $\chi^{2}$ is indicated. The dotted lines are the maximum and minimum amplitude of the visibility fluctuations as the snapshot is rotated. Bottom panel: closure phase (in degrees) versus the maximum projected baseline length for each baseline triplet. The observations (red dots with error bars) are fitted with the same intensity map and rotation angle used for the visibilities (black crosses). The axisymmetric case is represented by the solid black lines. The dotted lines are the maximum and minimum of the closure phase variation as the snapshot is rotated.

the initial guess (otherwise the numerical grid would be inappropriate). The average final radius is determined once the simulation has ended. Therefore, since the radius (and the effective temperature) cannot be tuned, the model is placed at some distance to provide the angular diameter that most closely matches the observations. Finally, within the error bars our model radius 


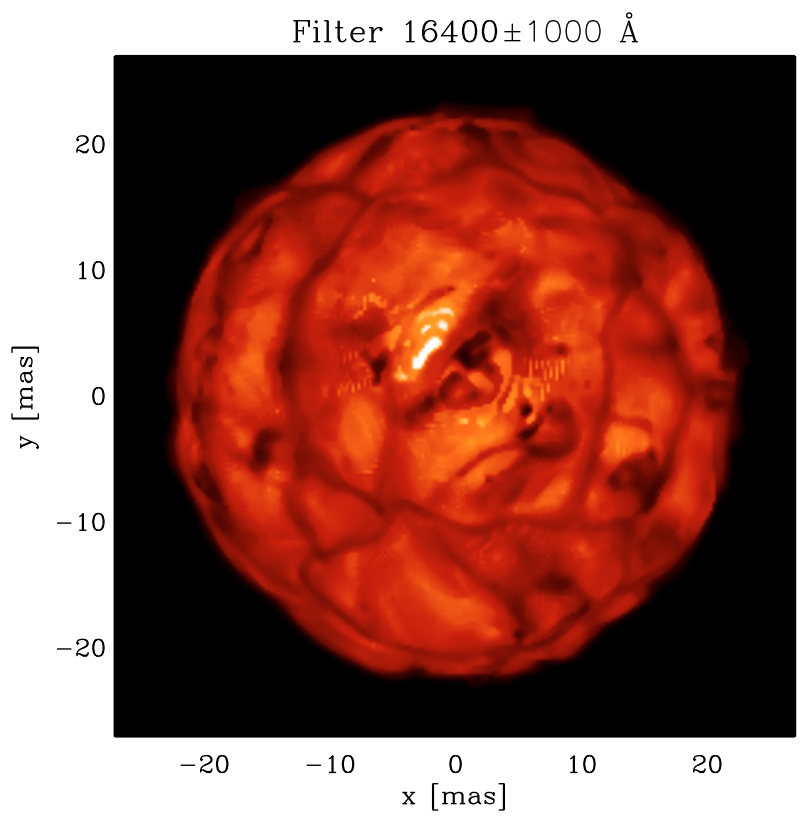

Fig. 3. Map of the linear intensity in the IONIC filter. The range is $\left[0 ; 3.1 \times 10^{5}\right] \mathrm{erg} \mathrm{cm}^{-2} \mathrm{~s}^{-1} \AA^{-1}$. The stellar parameters of this snapshot are: $L=92300 L_{\odot}, R=832.4 R_{\odot}, T_{\text {eff }}=3486 \mathrm{~K}$, and $\log (g)=-0.34$. The simulation has been scaled to an apparent diameter of $\sim 45.1$ mas, at a distance of $172.1 \mathrm{pc}$ to fit the data points in the first lobe.

agrees with all other data derived using the distance determined by Harper et al. (2008).

Our RHD simulation provides a more accurate fit of the data than uniform disk and limb-darkened models used by Haubois et al. in all lobes of the visibility function. The departure from circular symmetry is more evident at high spatial frequencies (e.g., the fourth lobe) where the visibility predicted from the parametric model is lower than the observed data. The smallscale convection-related surface structures are the cause of this departure and can only be explained by RHD simulations that are permeated with irregular convection-related structures of different size. The closure phases also display a good agreement with the simulation indicating that a possible solution to the distribution of the inhomogeneities on the surface of Betelgeuse is the intensity map of Fig. 3 (though the reconstructed images found by Haubois et al. (2009) are more probable).

This is the first robust confirmation of the physical origin of surface granulation for Betelgeuse, following on from the detection in the $K$ band (Paper I). Haubois et al. (2009) were able to reconstruct two images of Betelgeuse, using the data presented in this work, with two different image reconstruction algorithms. The image reconstructed with WISARD (Meimon et al. 2009, 2005) is displayed in Fig. 4 (left). Both images reconstructed in in Haubois et al. have two spots of unequal brightness located at roughly the same positions near the center of the stellar disk. One of these spots is half the stellar radius in size. Figure 4 compares the reconstructed image to our best-fit snapshot of Fig. 3. Fainter structures are visible in the synthetic image (right panel), while the reconstructed image (left panel) is dominated by two bright spots. Moreover, the larger spot visible in the reconstructed image is not present in our synthetic image, whereas there is good agreement in terms of location with the smaller spot located close to the center. However, it is possible that the synthetic map does not match exactly the location of the spots because it cannot perfectly reproduce the closure phase data.

\subsubsection{Molecular contribution to the visibility curves}

It is important to determine which molecular species contribute the most to the intensity absorption in the stellar atmosphere. For this purpose, we used the best fit snapshot of Fig. 3 and recomputed the intensity maps in the IONIC filter using only $\mathrm{CO}$, $\mathrm{CN}$, and $\mathrm{H}_{2} \mathrm{O}$ molecules, because they are the largest absorbers at these wavelengths (Fig. 1 of this work and Fig. 3 of Paper I). The intensity maps displayed in Fig. 5 of this paper (top row) should be compared to the original one in Fig. 3, which accounts for all the molecular and atomic lines. The surfaces of $\mathrm{CO}$ and $\mathrm{CN}$ maps clearly show the granulation pattern and are spot-free. However, the $\mathrm{H}_{2} \mathrm{O}$ map exhibits dark spots, which can also be identified in the original intensity map.

We calculated visibility curves from these molecular intensity maps using the same rotation angle used to generate the synthetic data in Fig. 2. Figure 5 (bottom row) shows that the $\mathrm{H}_{2} \mathrm{O}$ visibility is the closest to the original one at both low and high spatial frequencies. We conclude that: (i) in the first lobe, the $\mathrm{H}_{2} \mathrm{O}$ visibility is smaller than the $\mathrm{CO}$ and $\mathrm{CN}$ visibilities. Thus the radius of the star is dependent on the $\mathrm{H}_{2} \mathrm{O}$ contribution. (ii) At higher frequencies, only the $\mathrm{H}_{2} \mathrm{O}$ visibility can fit the observed data, whereas the $\mathrm{CO}$ and $\mathrm{CN}$ visibilities reproduce the data poorly.

\subsubsection{Size distribution on the stellar surface}

We also characterized the typical size distribution on the stellar surface using interferometric observables. The large range of spatial frequencies of the observation (between 12 to $95 \mathrm{arcsec}^{-1}$ ) is very well suited to this purpose.

Our aim was to visualize the energy within the signal as a function of spatial frequency. After the computation of the Fourier transform, FT, we obtained $\hat{I}(u, v)=F T[I(x, y)]$, where $I(u, v)$ is the most closely matching intensity map of Fig. 3. The resulting complex number $\hat{I}(u, v)$ was multiplied by low-pass and high-pass filters to extract the information from different spatial frequency ranges (corresponding to the visibility lobes). Finally, an inverse Fourier transform, $\overline{F T}$, was used to obtain the filtered image: $I_{\text {filtered }}(x, y)=\overline{F T}[\hat{I}(u, v) \cdot$ filter $]$.

Figure 6 (top left panel) shows the filtered image at spatial frequencies, $v$, corresponding to the first lobe. Since we filtered the signal at high spatial frequencies, the image appears blurry and seems to contain only information about the stellar radius. However, the top right panel displays the signal related to all the frequencies higher than the first lobe: in this image, we clearly do not detect the central convective cell of $\approx 30$ mas size $(60 \%$ of the stellar radius) visible in Fig. 3. Thus, the first lobe also carries information about the presence of large convective cells.

Figure 6 (bottom row) shows the second lobe with convection-related structures of $\approx 10-15$ mas, (30\% of the stellar radius), and the third and fourth lobes with structures smaller than 10 mas. We conclude that we can detect convection-related structures of different size using visibility measurements at the appropriate spatial frequencies. However, only imaging can definitively characterize the size of granules. A first step in this direction has been carried out in Berger et al. (2010) (to be submitted soon), where the image reconstruction algorithms have been tested using intensity maps from this RHD simulation. In the case of Betelgeuse, we have fitted its interferometric observables between 12 and $95 \operatorname{arcsec}^{-1}$ and thus inferred the presence of small to medium scale granules (5 to 15 mas) and a large convective cell ( $\approx 30$ mas). 

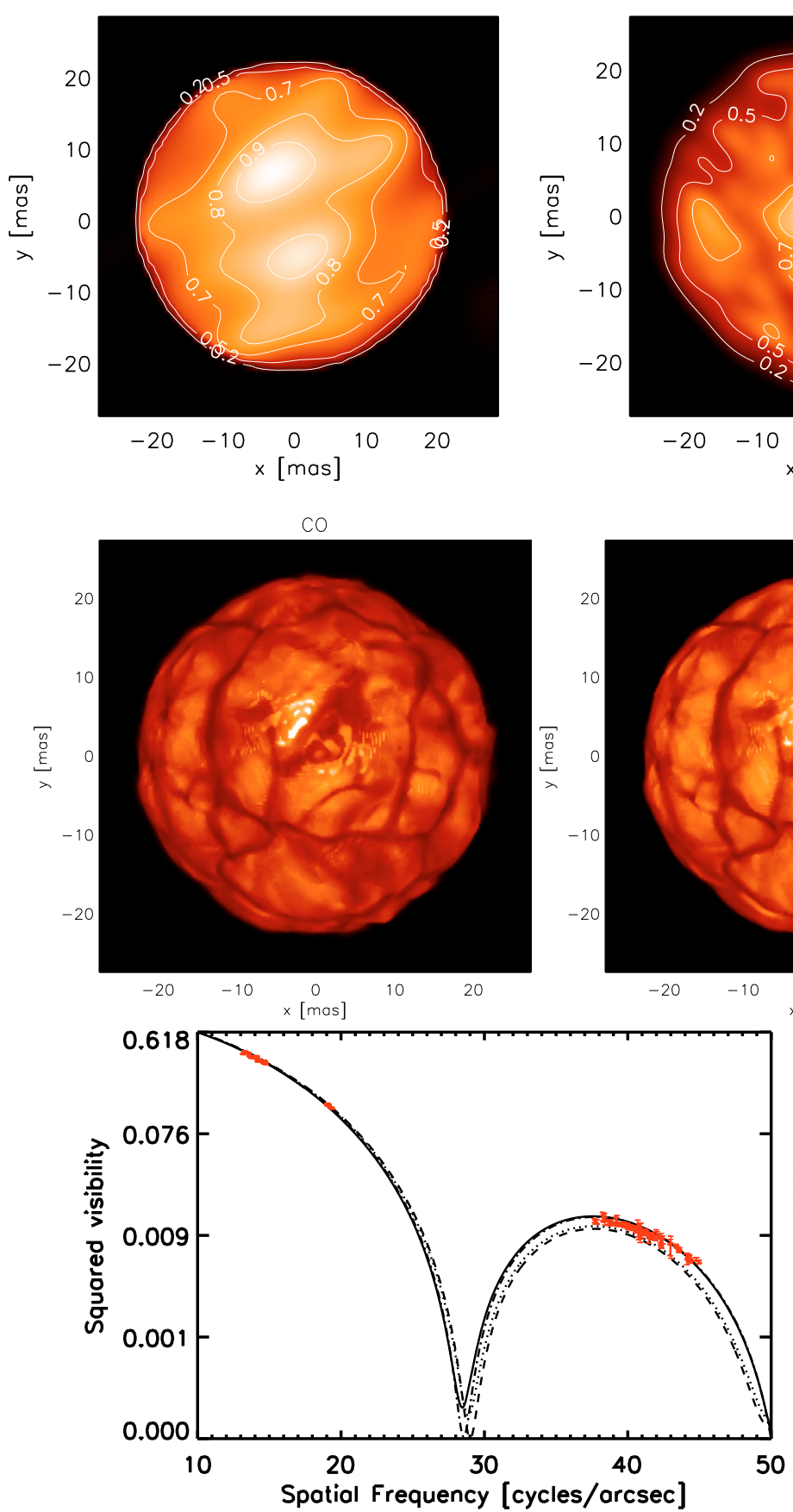

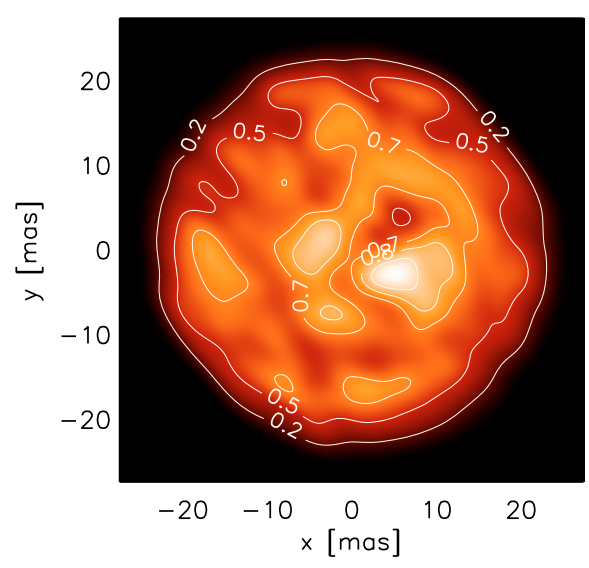

$\mathrm{CN}$
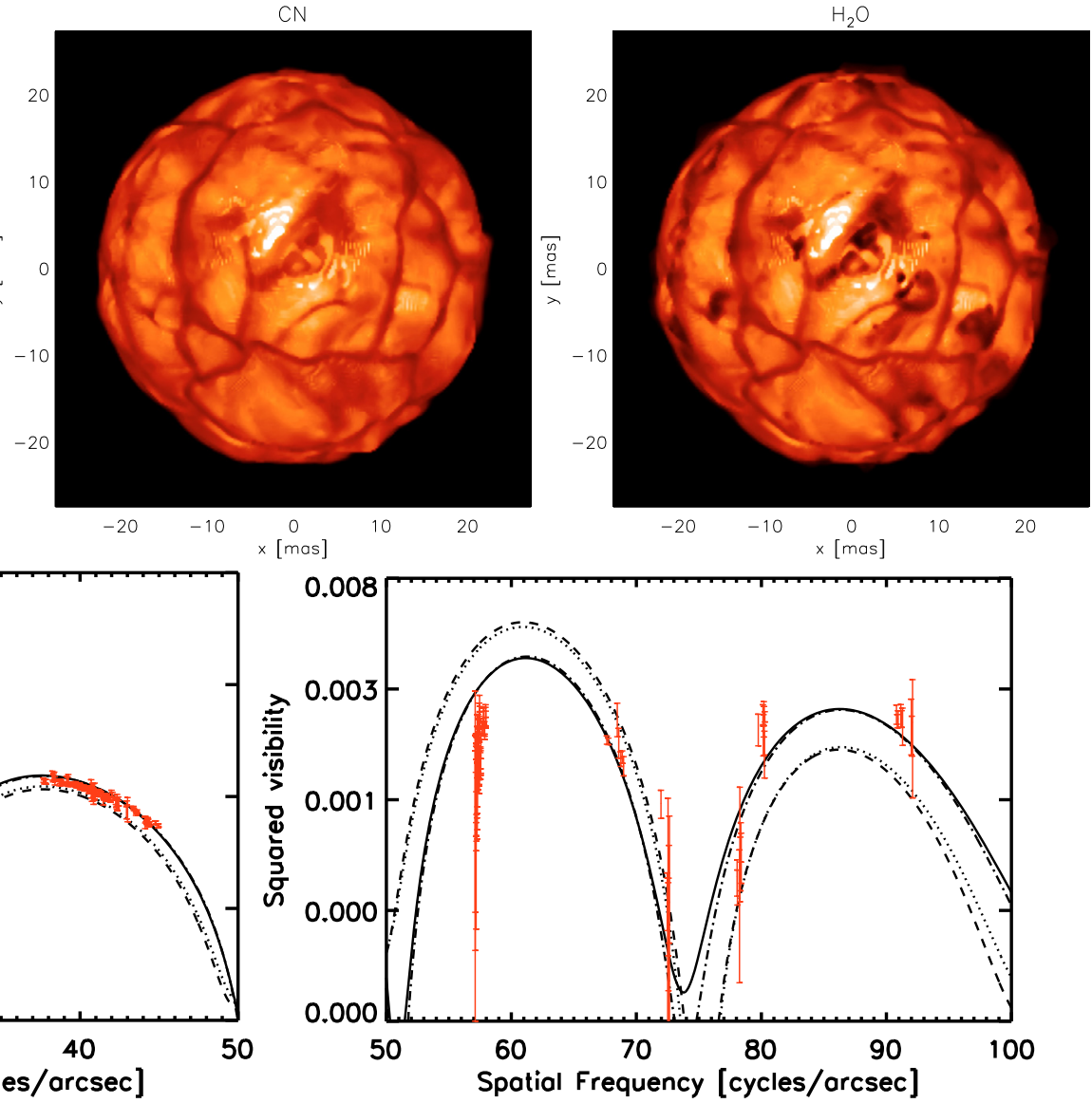

Fig. 4. Left panel: recontructed image from Haubois et al. (2009). Right panel: our bestfit 3D simulation snapshot of Fig. 3 convolved with a $6 \times 6$ mas PSF derived by fitting the central peak of the interferometric dirty beam. The intensities in both panels are normalized to the range $[0,1]$ and some contour lines are indicated $(0.2,0.5$, $0.7,0.8,0.9$ of the peak brightness).

Fig. 5. Top row: maps of the linear intensity in the IONIC filter of the molecular species (Fig. 1). The simulation snapshot and intensity range is the same as in Fig. 3. Bottom row: the most closely matching visibility curve (solid line) of Fig. 2 is compared to the visibility curve obtained from the intensity maps above: $\mathrm{CO}$ (dotted line), $\mathrm{CN}$ (dashed line), and $\mathrm{H}_{2} \mathrm{O}$ (dash dot line). Red data points are IOTA observations.

\subsection{Data from 7000 to $12900 \AA$}

The simulated surface in the optical to near-infrared region exhibits a spectacular pattern characterized by dark spots and bright areas. The brightest areas can be up to 50 times more intense than the dark ones. In addition, this pattern changes strongly with time and has a lifetime of a few weeks. In the wavelength region below $\approx 1 \mu \mathrm{m}$, the resulting surface pattern, though related to the granulation below, is also connected to dynamical effects. The light comes from higher up in the atmosphere where the optical depth is smaller than one and where waves and shocks begin to dominate. In addition to this, the emerging intensity depends on the variation in opacity through 

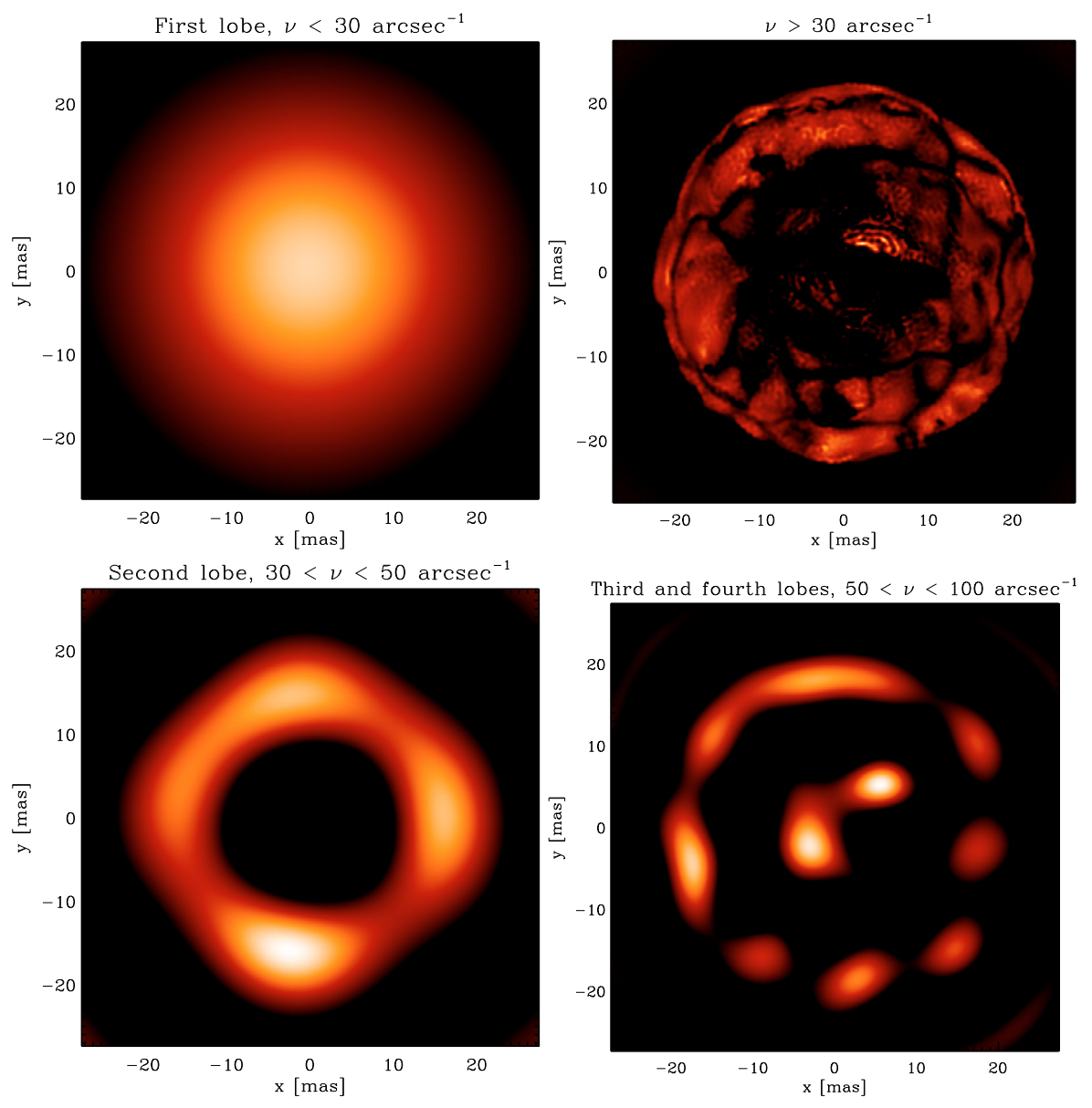

Fig. 6. Intensity maps filtered at different spatial frequencies corresponding to the lobes of the visibility curve shown in Fig. 2 . The images are normalized between $[0,1]$.

(Young et al. 2000), and (ii) the data from 2004 (Young et al. 2004). We proceeded as described in Sect. 4.1. We note that the filter curve for the $7820 \AA$ bandpass was lost and so a tophat function was assumed instead; we tested the validity of this assumption by replacing the known optical-region filter curves with top-hats, which did not affect the synthetic visibility and closure phase data significantly. Among the large number of computed visibilities and closure phases for each filter, we found that there are two snapshots of the simulated star, one for each epoch, that fit the observations. At each epoch, the same rotation angle of the snapshot was found to fit all of the observed wavebands.

Figure 7 displays the comparison to the data taken in 1997. The $7000 \AA$ synthetic image corresponds to a region with strong TiO absorption (transition $\mathrm{A}^{3} \Phi-\mathrm{X}^{3} \Delta(\gamma)$, see Fig. 1). This is also true for the $9050 \AA$ image (transition $\mathrm{E}^{3} \Pi-\mathrm{X}^{3} \Delta(\epsilon)$ ) but in this case the $\mathrm{TiO}$ band is weaker. The relative intensity of $\mathrm{TiO}$ bands depend on the temperature gradient of the model and change smoothly from one snapshot to another. The map at $12900 \AA$ is $\mathrm{TiO}$ free and detects mostly $\mathrm{CN}$ lines: in this case, the surface intensity contrast is less strong than in the TiO bands.

Figure 8 shows the comparison with the data taken in 2004. There, the filters used span wavelength regions corresponding to $\mathrm{TiO}$ absorption bands of different strengths centered on $7500 \AA, 7800 \AA$, and $9050 \AA$ (transitions $\mathrm{A}^{3} \Phi-\mathrm{X}^{3} \Delta(\gamma)$ and $\mathrm{E}^{3} \Pi-\mathrm{X}^{3} \Delta(\epsilon)$, see Fig. 1). Again, the same snapshot fitted the whole dataset from the same epoch.
The departure from circular symmetry is more evident than in the $H$ band, the first and the second lobes already showing large visibility fluctuations. The RHD simulation shows excellent agreement with the data in both the visibility curves and closure phases.

However, within the same observation epoch we had to scale the size of the simulated star to a different apparent diameter at each observed wavelength. For example, in Fig. 8 the apparent diameter varies from 47.3 to 52.6 mas. Thus, we infer that our RHD simulation fails to reproduce the $\mathrm{TiO}$ molecular band strengths probed by the three filters. As already pointed out in Paper I, our RHD simulations are constrained by execution time and they use a grey approximation for the radiative transfer that is well justified in the stellar interior and is a crude approximation in the optically thin layers; as a consequence, the thermal gradient is too shallow and weakens the contrast between strong and weak lines (Chiavassa et al. 2006). The intensity maps appear too sharp with respect to the observations. The implementation of non-grey opacities by employing five wavelength bins to describe the wavelength dependence of radiation fields (see Ludwig et al. 1994; Nordlund 1982, for details) should change the mean temperature structure and the temperature fluctuations. The mean thermal gradient in the outer layers, where $\mathrm{TiO}$ absorption has a large effect, should increase. In a subsequent step, the inclusion of the radiation pressure in the simulations should then lead to a different density/pressure structure with a less steep decline of density with radius. We expect the intensity maps probing $\mathrm{TiO}$ bands with different strengths to eventually 

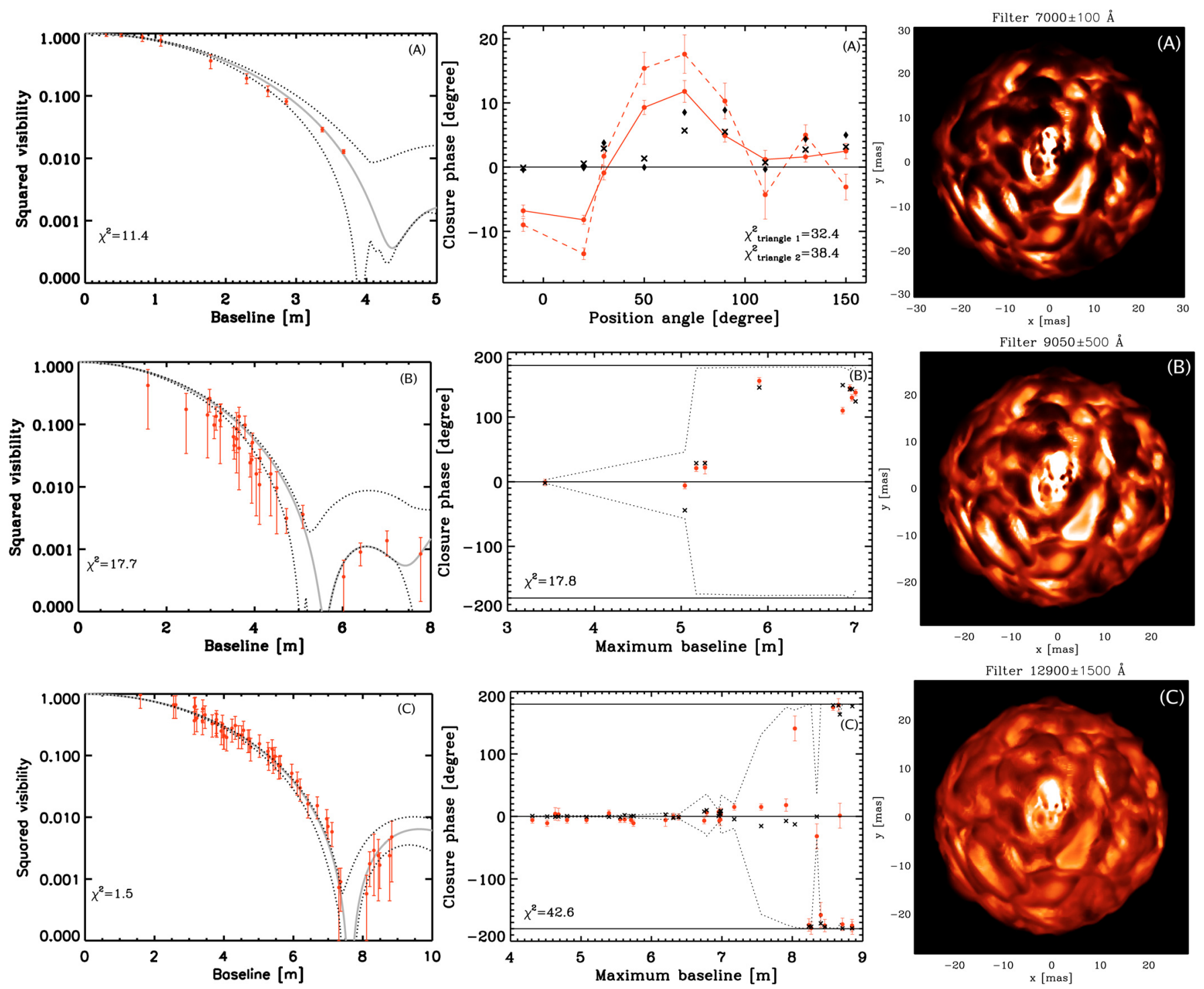

Fig. 7. Comparison to the data taken in 1997 (Young et al. 2000). The symbols in the visibility curves and closure phases plots have the same meaning as in Fig. 2. The intensity are normalized between $\left[0 ; 3.5 \times 10^{5}\right] \mathrm{erg} \mathrm{cm}^{-2} \mathrm{~s}^{-1} \AA^{-1}$. The stellar parameters of this snapshot are: $L=$ $93500 L_{\odot}, R=843.3 R_{\odot}, T_{\text {eff }}=3475 \mathrm{~K}$ and $\log (g)=-0.35$. The row marked with (A), (B) and (C) correspond to bandpass centered on 7000, 9050 and $12900 \AA$, respectively. The visibility curves are plotted in left column, while the closure phases are shown in central column. The intensity maps centered on 7000 and $9050 \AA$ have been scaled to an apparent diameter of $\sim 48.9$ mas, at a distance of 161.1 pc fitting the data points in the first lobe; the map at $12900 \AA$ to an apparent diameter of $\sim 47.1$ mas, at a distance of 167.1 pc. Row marked with (A): the observed visibility amplitudes (90 visibilities) have been averaged over the position angle of the aperture mask for the sake of clarity. We performed the fitting procedure for each of the unaveraged visibilities, and then we plot the averaged value. The closure phases of two (triangle 1 in solid and triangle 2 in dashed red line) of the 10 observed triangles have been plotted as a function of the position angle of the aperture mask on the sky. The best-matching synthetic closure phases are black crosses for triangle 1 and black diamonds for triangle 2 .

show larger diameter variations due to the molecular absorption as a result of these refinements.

Young et al. (2000) managed to model the data in the $7000 \AA$ filter with two best-fit parametric models, consisting of a circular disk with superimposed bright features (Fig. 9, central panel) or dark features (left panel). We compared these parametric models to our best-fit synthetic image of Fig. 7 (top row). The convolved image, displayed in Fig. 9 (right panel), shows a closer qualitative agreement with the bright features parametric model. The 3D simulations show that the surface contrast is enhanced by the presence of significant molecular absorbers such as $\mathrm{TiO}$ which contribute in layers where waves and shocks start to dominate. The location of bright spots is then a consequence of the underlying activity.

Page 8 of 10

\section{Conclusions}

We have used radiation hydrodynamical simulations of red supergiant stars to explain interferometric observations of Betelgeuse from the optical to the infrared region.

The picture of the surface of Betelgeuse portrayed by our study is the following: (i) a granulation pattern is undoubtedly present on the surface and the convection-related structures have strong signatures in the visibility curve and closure phases at high spatial frequencies in the $H$ band and on the first and second lobes in the optical region. (ii) In the $H$ band, Betelgeuse is characterized by a granulation pattern composed of convection-related structures of different sizes, including small to medium scale granules (5-15 mas) and a large convective cell 

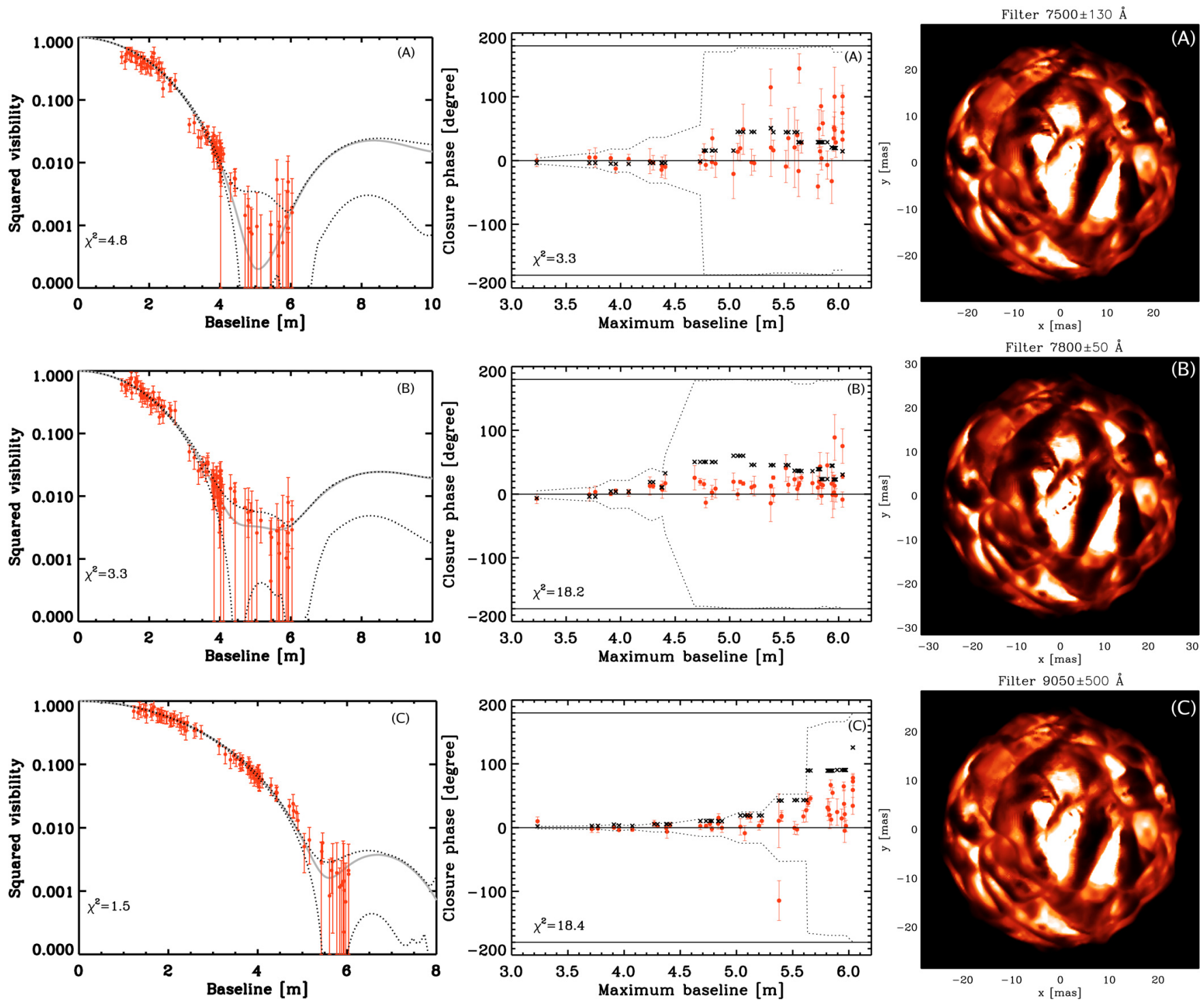

Fig. 8. Same as in Fig. 7 but for the observations of Young et al. (2004). The stellar parameters of this snapshot are: $L=93800 L_{\odot}, R=840.3 R_{\odot}$, $T_{\text {eff }}=3484 \mathrm{~K}$ and $\log (g)=-0.35$. The row marked with (A), (B), and (C) correspond to bandpass centered on 7500,7820 and $9050 \AA$, respectively. The intensity map centered at $7500 \AA$ has been scaled to an apparent diameter of $\sim 49.7$ mas, at a distance of 157.9 pc; the map at $7820 \AA$ to an apparent diameter of $\sim 47.3$ mas, at a distance of $165.7 \mathrm{pc}$; and the map 9050 to an apparent diameter of $\sim 52.6$ mas, at a distance of $149.1 \mathrm{pc}$.
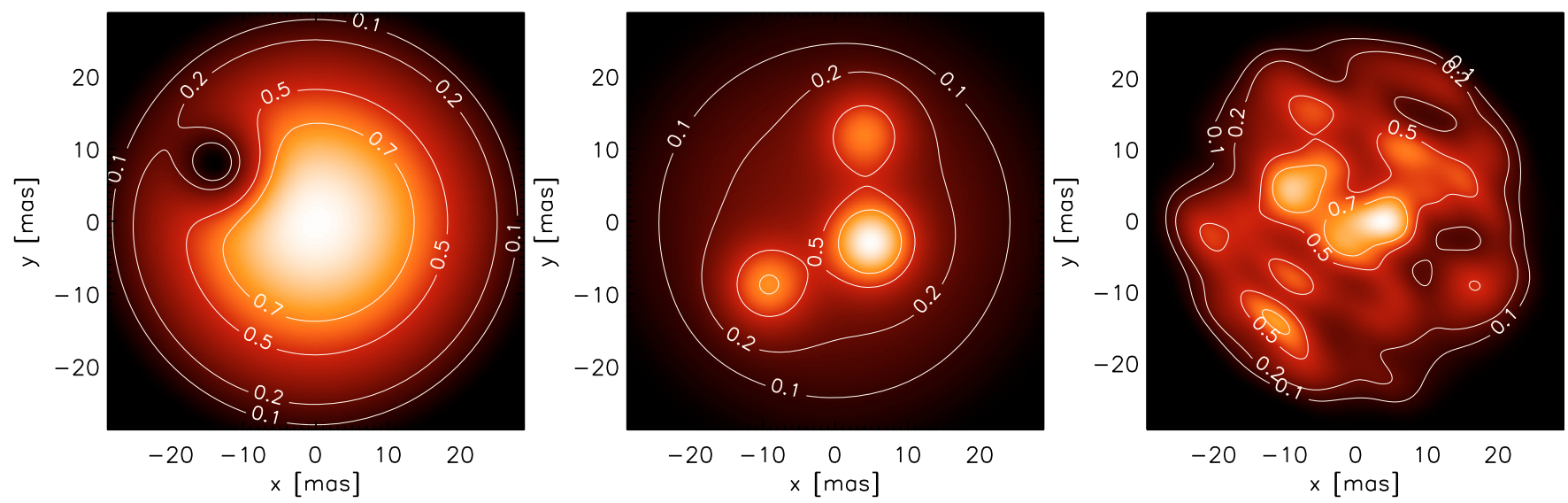

Fig. 9. Left and central panels: brightness distributions of the best fitting parametric models from Young et al. (2000) with dark features (left) and bright features (center). Right panel: our best fitting 3D simulation snapshot of Fig. 7 (row marked with (A)) convolved with a $10 \times 10$ mas PSF (i.e., the size of the bright features in the parametric model). The intensities in both panels are normalized to the range $[0,1]$ and some contour lines are indicated $(0.1,0.2,0.5,0.7)$. 
( $\approx 30$ mas). This supports previous detections carried out using RHD simulation in the $K$ band (Paper I), for parametric models and the same dataset (Haubois et al. 2009); Kervella et al. (2009) with VLT/NACO observations; and Ohnaka et al. (2009) with VLTI/AMBER observations. We have demonstrated that $\mathrm{H}_{2} \mathrm{O}$ molecules contribute more than $\mathrm{CO}$ and $\mathrm{CN}$ to the position of the visibility curve's first null (and thus to the measured stellar radius) and to the small-scale surface structures. (iii) In the optical, Betelgeuse's surface appears more complex with areas up to 50 times brighter than dark areas. This is indicative of the underlying activity characterized by interactions between shock waves and non-radial pulsations in layers where there are strong TiO molecular bands.

These observations provide a wealth of information about both the stars and our RHD models. The comparison with the observations in the $\mathrm{TiO}$ bands allowed us to suggest which approximations must be replaced with more realistic treatments in the simulations. New models of higher wavelength resolution (i.e., non-grey opacities) are being developed and will be tested against these observations. From the observational point of view, additional multi-epoch observations, in both the optical and the infrared, are needed to assess the time variability of convection.

Acknowledgements. This project was supported by the French Ministry of Higher Education through an ACI (PhD fellowship of Andrea Chiavassa, postdoctoral fellowship of Bernd Freytag, and computational resources). Present support is ensured by a grant from ANR (ANR-06-BLAN-0105). We are also grateful to the PNPS and CNRS for its financial support through the years. We thank the CINES for providing some of the computational resources necessary for this work.

\section{References}

Asplund, M., Grevesse, N., \& Sauval, A. J. 2006, Commun. Asteroseismol., 147, 76

Baldwin, J. E., Boysen, R. C., Cox, G. C., et al. 1994, in SPIE Conf. Ser. 2200, ed. J. B. Breckinridge, 118

Baldwin, J. E., Haniff, C. A., Mackay, C. D., \& Warner, P. J. 1986, Nature, 320, 595

Berger, J.-P., Haguenauer, P., Kern, P. Y., et al. 2003, in Presented at the Society of Photo-Optical Instrumentation Engineers (SPIE) Conf., Interferometry for Optical Astronomy II, ed. W. A. Traub, Proc. SPIE, 4838, 1099

Burns, D., Baldwin, J. E., Boysen, R. C., et al. 1997, MNRAS, 290, L11
Buscher, D. F., Baldwin, J. E., Warner, P. J., \& Haniff, C. A. 1990, MNRAS, 245, $7 \mathrm{P}$

Chiavassa, A., Plez, B., Josselin, E., \& Freytag, B. 2006, in SF2A-2006: Semaine de l'Astrophysique Francaise, ed. D. Barret, F. Casoli, G. Lagache, A. Lecavelier, \& L. Pagani , 455

Chiavassa, A., Plez, B., Josselin, E., \& Freytag, B. 2009, A\&A, 506, 1351

Freytag, B. 2003, in Interferometry for Optical Astronomy II, ed. W. A. Traub, Proc. SPIE Conf., 4838, 348

Freytag, B., \& Höfner, S. 2008, A\&A, 483, 571

Freytag, B., Steffen, M., \& Dorch, B. 2002, Astron. Nachr., 323, 213

Gray, D. F. 2008, AJ, 135, 1450

Gustafsson, B., Bell, R. A., Eriksson, K., \& Nordlund, A. 1975, A\&A, 42, 407 Gustafsson, B., Edvardsson, B., Eriksson, K., et al. 2003, in Stellar Atmosphere

Modeling, ed. I. Hubeny, D. Mihalas, \& K. Werner, ASP Conf. Ser., 288, 331 Gustafsson, B., Edvardsson, B., Eriksson, K., et al. 2008, A\&A, 486, 951 Haniff, C. A., Mackay, C. D., Titterington, D. J., et al. 1987, Nature, 328, 694 Harper, G. M., Brown, A., \& Guinan, E. F. 2008, AJ, 135, 1430

Haubois, X., Perrin, G., Lacour, S., et al. 2009, A\&A, 508, 923 Josselin, E., \& Plez, B. 2007, A\&A, 469, 671

Kervella, P., Verhoelst, T., Ridgway, S. T., et al. 2009, A\&A, 504, 115 Kiss, L. L., Szabó, G. M., \& Bedding, T. R. 2006, MNRAS, 372, 1721 Levesque, E. M., Massey, P., Olsen, K. A. G., et al. 2005, ApJ, 628, 973 Levesque, E. M., Massey, P., Olsen, K. A. G., et al. 2006, ApJ, 645, 1102 Ludwig, H.-G., Jordan, S., \& Steffen, M. 1994, A\&A, 284, 105

Meimon, S., Mugnier, L. M., \& Le Besnerais, G. 2005, J. Opt. Soc. Am. A, 22, 2348

Meimon, S., Mugnier, L. M., \& Le Besnerais, G. 2009, J. Opt. Soc. Am. A, 26, 108

Meynet, G., \& Maeder, A. 2003, A\&A, 404, 975

Monnier, J. D., Traub, W. A., Schloerb, F. P., et al. 2004, ApJ, 602, L57

Nordlund, A. 1982, A\&A, 107, 1

Ohnaka, K., Hofmann, K., Benisty, M., et al. 2009, A\&A, 503, 183

Pedretti, E., Millan-Gabet, R., Monnier, J. D., et al. 2004, PASP, 116, 377

Perrin, G., Ridgway, S. T., Coudé du Foresto, V., et al. 2004, A\&A, 418, 675

Tatebe, K., Chandler, A. A., Wishnow, E. H., Hale, D. D. S., \& Townes, C. H. 2007, ApJ, 670, L21

Traub, W. A., Ahearn, A., Carleton, N. P., et al. 2003, in SPIE Conf. Ser. 4838, ed. W. A. Traub, 45

Tuthill, P. G., Haniff, C. A., \& Baldwin, J. E. 1997, MNRAS, 285, 529

Uitenbroek, H., Dupree, A. K., \& Gilliland, R. L. 1998, AJ, 116, 2501

Wilson, R. W., Baldwin, J. E., Buscher, D. F., \& Warner, P. J. 1992, MNRAS, 257, 369

Wilson, R. W., Dhillon, V. S., \& Haniff, C. A. 1997, MNRAS, 291, 819

Young, J. S., Badsen, A. G., Bharmal, N. A., et al. 2004

Young, J. S., Baldwin, J. E., Beckett, M. G., et al. 1998, in SPIE Conf. Ser. 3350, ed. R. D. Reasenberg, 746

Young, J. S., Baldwin, J. E., Boysen, R. C., et al. 2000, MNRAS, 315, 635

Zhao, M., Monnier, J. D., Torres, G., et al. 2007, ApJ, 659, 626 